\title{
An Online Condition Monitoring Thermal Prognostic Indicator System for MV Cable Circuits
}

\author{
S. Christou, P. L. Lewin, J. A. Pilgrim and S. G. Swingler \\ School of Electronics and Computer Science \\ University of Southampton \\ Southampton, United Kingdom \\ e-mail: sc10g09@soton.ac.uk
}

\begin{abstract}
Developing a reliable online condition monitoring prognostic indicator tool for $\mathrm{MV}$ cables is of great importance as it can predict and prevent upcoming failures of the distribution cable circuits. This paper introduces a thermal prognostic model for MV underground cable terminations based on a support vector regression algorithm. The model is shown to predict the likely temperature along the cable thirty minutes into the future and is able to rapidly identify temperature anomalies which may indicate upcoming failures.
\end{abstract}

Keywords-Condition Monitoring; Support Vector Regression; Diagnostic; Prognostic; Insulation System.

\section{INTRODUCTION}

The ability to accurately monitor the real-time health of power network systems is the ultimate aim for utility companies for tasks such as planning, asset management and identifying possible weaknesses within their networks.

The power network distributors aim to supply electricity as safely and reliably as possible. Nevertheless failures in the power distribution networks are still regular occurrences [1]. The most common location of cable failures are at the cable joints or terminations $[2,3]$. Over the past years there have been a number of failures reported within cable terminations, in particular near the termination sealing ends, where in some cases the monitored temperatures increase by up to $10^{\circ} \mathrm{C} \quad$ [4$6]$.

This paper presents a method of developing a reliable thermal prognostic indicator system for cable terminations. An experiment has been set up where a closed loop $33 \mathrm{kV}$ cable is tested under current loading patterns. Real-time measurements (air ambient temperature, temperature of the cable terminations and loading demand) taken close to the cable are used to update the prognostic simulation model based on the support vector regression (SVR) algorithm. The model predicts the likely temperatures of cable terminations 30 minutes into the future. Anomalies of the measurements along the cable are compared with predicted values in order to identify a possible degradation activity in the cable terminations.

It is assumed that an increase of local cable temperature, i.e. a hot-spot, is indicative of degradation of the cable insulation due to thermomechanical, electrical and environmental factors.

This work is supported by the Research Councils UK, through the HubNet consortium, www.hubnet.org.uk (grant number: EP/I013636/1)

\section{EXPERIMENTAL SETUP}

A closed loop of 10 meters $33 \mathrm{kV}$ cable experiment, under different current loading patterns, was used to generate data needed to develop the thermal prognostic model. Two ends of the cable are electrically connected to create a loop. As the cable circuit length is short a single-point-bonding is used to avoid any sheath circulating current on the cable.

Two current transformers (CT1 and CT2) and a motorized variac unit are used to generate the current loading profiles. A control system program was developed in order to vary the input current to the CTs and to simulate different load profiles. A data acquisition (DAQ) device collaborates with the control system program by receiving and sending analog signals. A Rogowski coil is used to measure the current passing through the cable conductor in real-time. This current is input to the DAQ which updates the control system program. Afterwards the control system program sends the appropriate signals to the motorized variac and adjusts the corresponding load profile demand in the cable to be close to the expected current value via the DAQ.

The surface temperature of the cable was continuously monitored using 7 thermocouples ( $\mathrm{T} \#$, where \# is the thermocouple number) placed on the cable at various positions as is shown in Fig. 1. Three thermocouples (T1, T2 and T3) are located at the termination section. The other four thermocouples (T4, T5, T6 and T7) are located 2 meters away from each other. The thermocouples are connected to the datalogger which records the temperatures every minute and provides an average value of local temperature at 30 minute intervals.

During this experiment a daily load current cycle, as shown in Fig. 2, was loaded in the $33 \mathrm{kV}$ cable producing an increase in temperature on the surface of the cable. The experiment was run for two weeks, with the same current load. The first week of the experiment was used to gather data in order to build the thermal prognostic model while the second week was used to test the performance of the model. During the second week of loading, artificial hot-spots were introduced to the cable by adding fiberglass insulation sections. The fiberglass sections act to increase the local thermal resistance at the cable surface. The additional hotspots allow investigation of whether the thermal prognostic simulation model is sufficiently sensitive to detect temperature 
anomalies at the cable surface. The two thermal insulation sections were added close to the end of the termination, where failures are more likely to occur. The first insulation was added by the end of the termination, located 0.10 meters from $\mathrm{T} 2$ and $0.35 \mathrm{~m}$ from $\mathrm{T} 1$. The second insulation was added above the T3, 0.10 meters away from the end section of the termination.

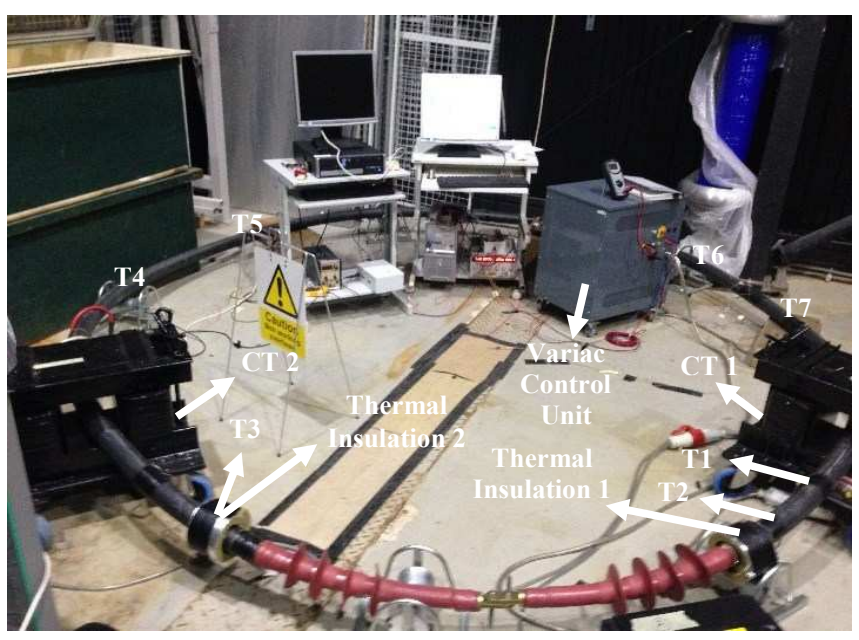

Fig. 1 Experimental setup of $33 \mathrm{kV}$ cable.

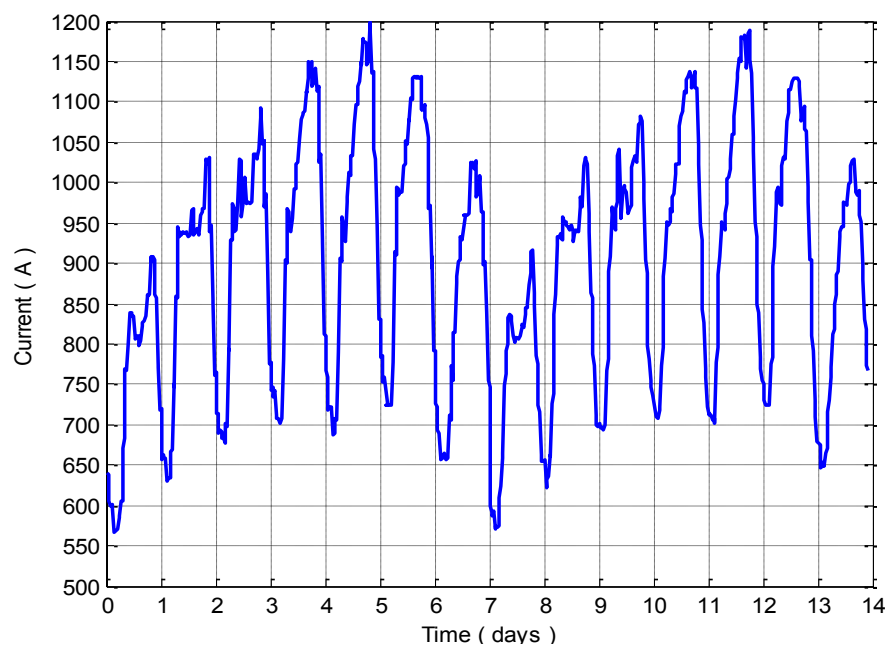

Fig. 2 Experimental daily loading profile.

\section{THERMAL PROGNOSTIC SIMULATION MODEL BASED ON SVR}

The SVR is a machine learning algorithm which aims to determine an approximation function, from a training dataset, that has a maximum pre define deviation from the targets output values and at the same time is not complex. It always perform a linear regression by transforming the input data features into a high dimensional feature space through a nonlinear mapping [7]. SVR is able to avoid overfitting issues and creates less complex models that always converge to a solution [8]. The thermal prognostic model was developed using the LIBSVM toolbox [9] in Matlab.

\section{A. Data Preparation}

The input data needed for the development of the SVR are shown in Table I.

TABLE I INPUT DATA FOR SVR

\begin{tabular}{|c|c|c|}
\hline $\begin{array}{c}\text { Input } \\
\text { Columns }\end{array}$ & Features & Description \\
\hline $1-4$ & $\begin{array}{c}\text { Air ambient } \\
\text { temperature }\end{array}$ & $\begin{array}{c}\text { Mean air temperature captured } \\
\text { every 30 minutes }\end{array}$ \\
\hline $5-8$ & Current loading & $\begin{array}{c}\text { Mean current loading captured } \\
\text { every 30 minutes }\end{array}$ \\
\hline $9-12$ & T5 & $\begin{array}{c}\text { Temperature of T5 captured every } \\
30 \text { minutes }\end{array}$ \\
\hline
\end{tabular}

T5 is used as an input variable for SVR training as well as the air ambient temperature in the laboratory and the current load profile of the experiment. The number of input columns correspond to 2 hours of input data. Each 2 hour interval contains patterns that the SVR uses to build the relationship and the generalization function for the input feature data against the output data.

The 30 minutes prediction horizon proved to be the most suitable prediction time when considering the thermal time constant of a loaded MV cable. In addition as the data are averaged every 30 minutes only 1 data point needs to be predicted by the SVR.

In order to avoid features with larger numeric values being dominant to those with smaller values, input data has to be scaled before being fed into the SVR. Scaling of the input data reduces computation time during the modelling process. In this model the data were scaled from $[0,1]$ as follows:

$$
X \text { scale }=\frac{X i-X \min }{X \max -X \min }
$$

where $X$ is the original data, $X$ scale the scaling data, $X \max$ and $X$ min are the maximum and minimum values of $X$.

\section{B. Selection of SVR parameters}

Selection of the appropriate SVR tunable parameters $C, \gamma$ and $\varepsilon$ plays a significant role to achieve a good generalization performance. The parameter $\mathrm{C}$ controls the trade-off between model complexity and the frequency with which errors are allowed. The parameter $\gamma$ controls the width of the GaussianRBF kernel which is used to map the input features to a higher dimensional space. The combination of $\mathrm{C}$ and $\gamma$ controls the input range of the training data and therefore these two values have to be tuned together. The parameter $\varepsilon$ is associated with the deviation of the output value from the training data. Larger deviation value of $\varepsilon$ will result in less complex underfitting model. On the other hand small value of $\varepsilon$ will result in more complex overfitting model. The temperature data collected during the experiment has a precision of two decimal places hence the value of $\varepsilon=0.01$ has been selected. The kernel selection as well as the grid-search technique and crossvalidation method used to identify the best pair of $C$ and $\gamma$ can be found in a previously reported work [10]. 


\section{EXPERIMENTAL RESULTS}

Three different models were built to detect temperature increases at T1, T2 and T3 after the introduction of the hotspots produced by the fiberglass insulation sections by the end of the termination.

The hot-spots were introduced on the second week of the experiment. The first 5 days of the experiment were used to train the SVR algorithm, the next 2 days to test its performance using root mean square error (RMSE) and to identify the best combination of $\mathrm{C}$ and $\gamma$. Finally in the last week the models, developed the week before, were left to run without any change of parameters under unknown conditions and under the influence of local hot-spots.

Figures 4, 5 and 6 show 30 minutes ahead predictions made for the testing and unknown data for T1, T2 and T3 without and with the influence of hot-spots.

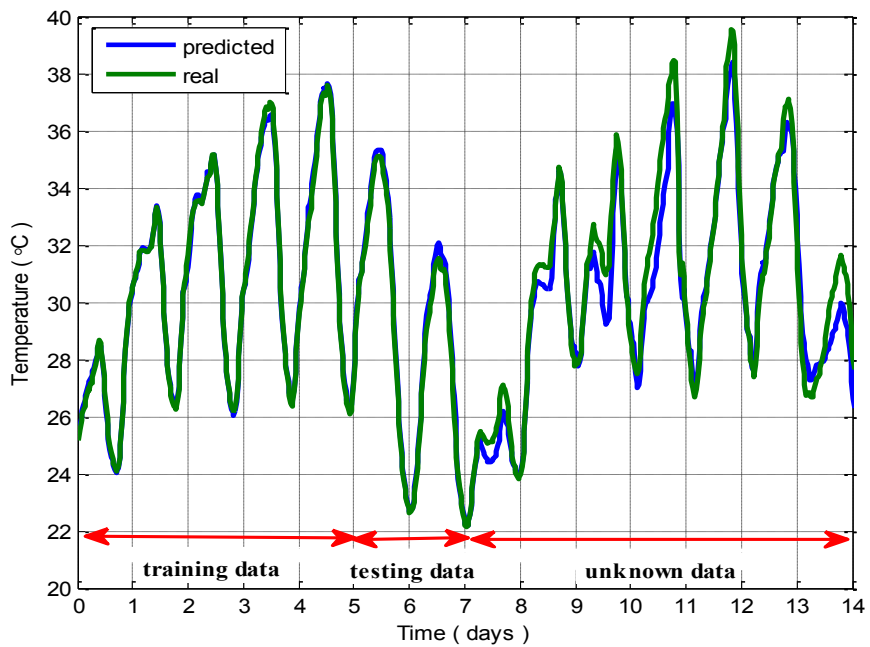

Fig. 4 Comparison between predicted temperature on testing data $(\mathrm{RMSE}=1.11 \%)$ and unknown data $(\mathrm{RMSE}=3.50 \%)$ with the real temperature of $\mathrm{T} 1$.

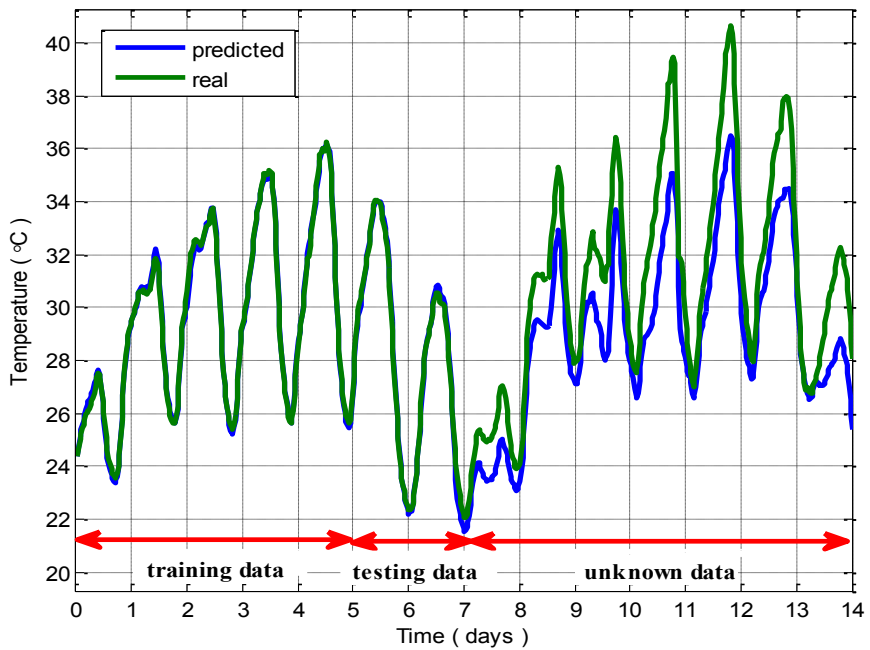

Fig. 5 Comparison between predicted temperature on testing data $(\mathrm{RMSE}=0.89 \%)$ and unknown data $(\mathrm{RMSE}=7.71 \%)$ with the real temperature of $\mathrm{T} 2$.

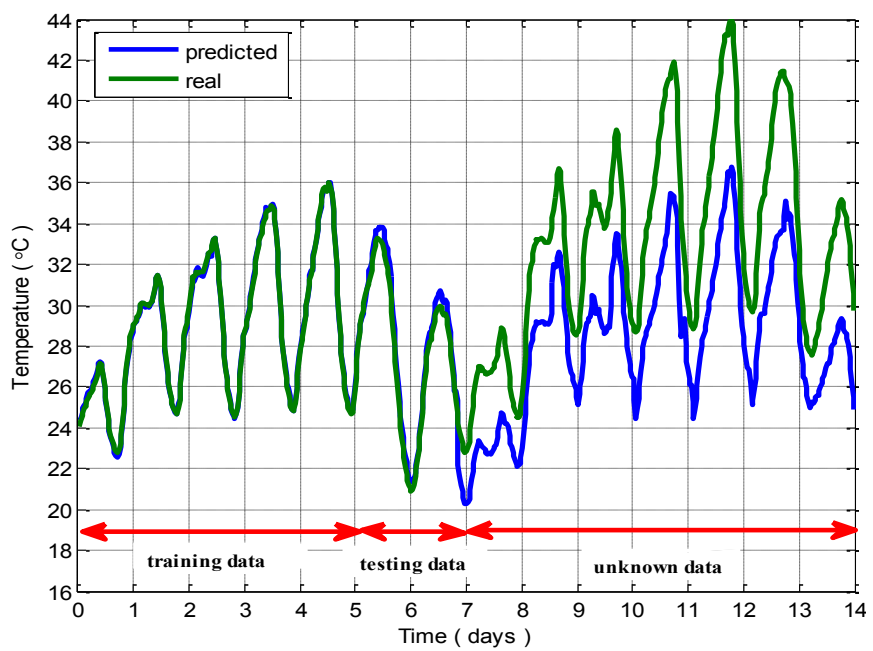

Fig. 6 Comparison between predicted temperature on testing data $(\mathrm{RMSE}=1.26 \%)$ and unknown data $(\mathrm{RMSE}=14.46 \%)$ with the real temperature of $\mathrm{T} 3$.

The prediction made for the testing data of T1, T2 and T3 shows good results with prediction RMSE less than $1.3 \%$ as can been seen from Figures 4, 5 and 6. After the introduction of the hot-spots from the day 7 until day 14 of the experiment, the RMSE for T1, T2 and T3 increased to $3.50 \%, 7.71 \%$ and $14.46 \%$ respectively. It can be observed that all of the thermocouples were able to identify the increase of temperature caused by the nearby hot-spots.

The thermal prognostic model has an accuracy of $\pm 1^{\circ} \mathrm{C}$. Hence in the event when the real temperature is higher than predicted temperature by at least $1{ }^{\circ} \mathrm{C}$, the positive part of the temperature prediction error graphs can be assumed to be an indication of an upcoming failure of the system.

Figures 7,8 and 9 show the temperature difference, real minus predicted, for the same thermocouples while the hotspots were disabled and enabled.

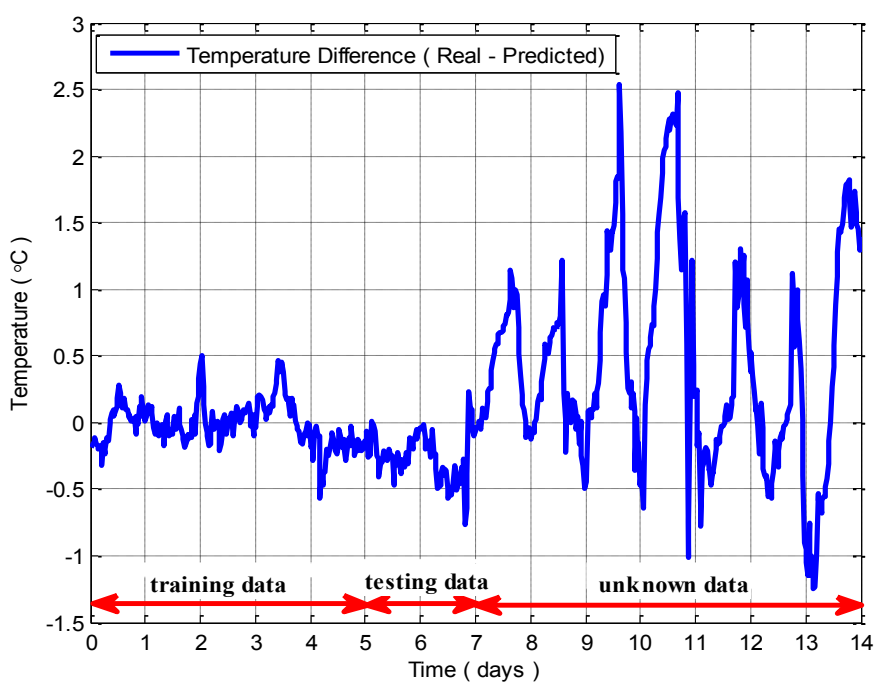

Fig. 7 Temperature prediction error for T1. 


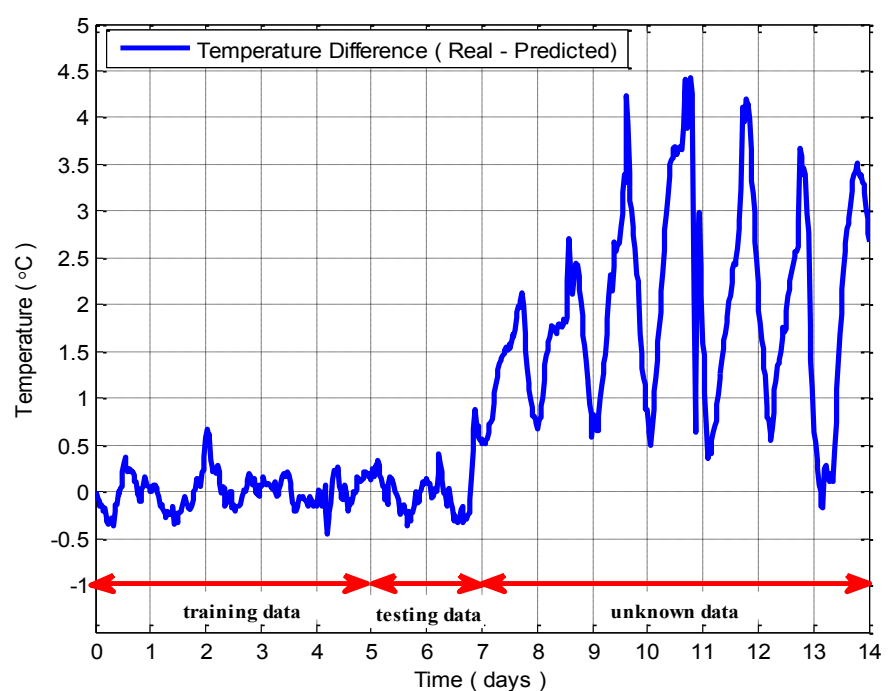

Fig. 8 Temperature prediction error for T2.

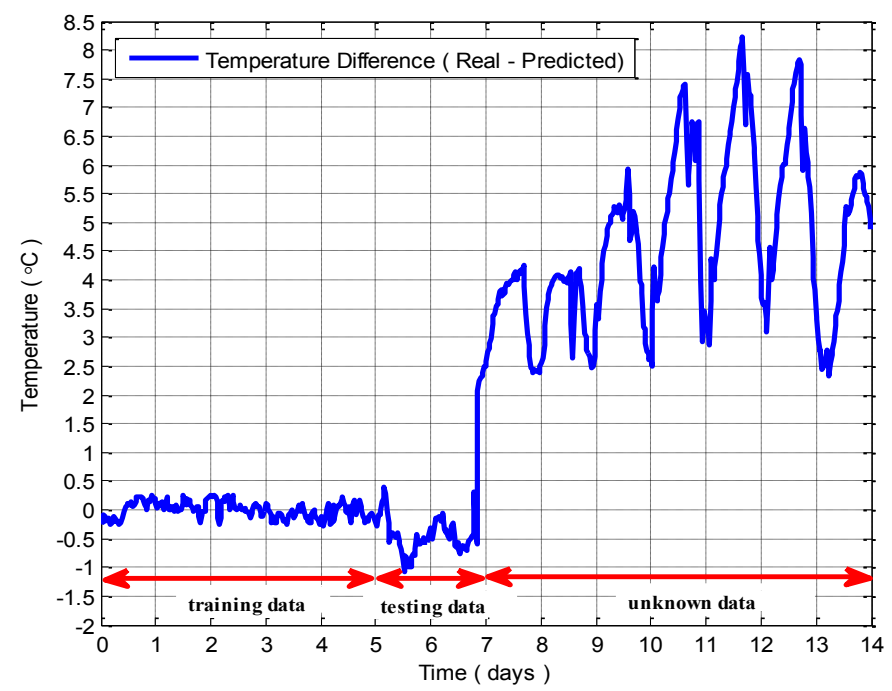

Fig. 9 Temperature prediction error for T3.

$\mathrm{T} 2$ which is $0.10 \mathrm{~m}$ away from the first hotspot, thermal insulation 1, identified an increase of temperature of the order of $4.42{ }^{\circ} \mathrm{C}$ while $\mathrm{T} 1$ which is located further away from the hot-spot, at the distance of $0.35 \mathrm{~m}$, could as well identify an increase of temperature of $2.54^{\circ} \mathrm{C}$. T3 which is located just below the second hot-spot, thermal insulation 2, had a temperature increase which reached $8.22^{\circ} \mathrm{C}$. The temperature prediction error for $\mathrm{T} 2$ and $\mathrm{T} 3$ is well above $1{ }^{\circ} \mathrm{C}$ as these thermocouples are located near or below the hot-spots. T1 positioned $0.35 \mathrm{~m}$ away from the location of the first hot-spot was able to identify the presence of hot-spots with the highest temperature magnitude falling during the daytime but could not detect the hotspots during the nighttime due to the much lower air ambient temperature.

\section{CONCLUSION}

This paper describes the development of a new thermal prognostic model based on SVR which can detect the temperature anomalies in the cable terminations using a prediction of temperatures 30 minutes into the future. The developed thermal model is able to monitor specific areas of a cable, such as terminations, where hot-spots are more likely to occur, and to identify the signs of imminent degradation in the cable.

Based on the experimental results presented, it can be concluded that the most effective distance for the thermal prognostic simulation model to identify the location of the hot-spots is within a radius of up to $0.35 \mathrm{~m}$. A distribution of thermocouples close to the area of interest is sufficient for the model to identify and predict a possible failure process at an early stage.

The use of such systems enables a form way of prognostic condition monitoring in with the aim to measure less and model more. Prognostic models will enable the power network operators to increase asset utilization and reduce costs.

\section{ACKNOWLEDGMENT}

The authors gratefully acknowledge the financial support of this work by the Research Councils UK, through the HubNet consortium, www.hubnet.org.uk (grant number: $\mathrm{EP} / \mathrm{I} 013636 / 1)$

\section{REFERENCES}

[1] H. Liu, Y. Bai, M. Liang, H. Chen, and X. Ren, "Research of state monitoring and pre-warning system of cable joint in distribution power grids," Int. Conf. Power Syst. Technol. POWERCON 2006, pp. 1-5, October 2006.

[2] R.N. Hampton, R.A. Hartlein, J. Perkel, M. Begovic, J.C. Hernandez, and Y. Del Valle, "Diagnostic Testing of Underground Cable Systems" (Cable Diagnostic Focused Initiative) - Final Report DOE Award No. DE-FC02-04CH11237, pp 1-328, 2011.

[3] K. Uchida, S. Kobayashi, T. Kawashima, H. Tanaka, S. Sakuma, K. Hirotsu, and H. Inoue, "Study on detection for the defects of XLPE cable lines," IEEE Trans. Power Deliv., vol. 11, no. 2, pp. 663-668, April 1996.

[4] C.Y. Choo and F. A. Auditoré;, "Strategic Replacement of $220 \mathrm{kV}$ Cable at Taranaki Combined Cycle Station," Electricity Engineers Association Conf., June 2010.

[5] U. Patel, S. H. Jayaram, A. El-Hag, and R. Seetahpathy, "MV cable termination failure assessment in the context of increased use of power electronics," Electr. Insul. Conf. EIC, pp. 418-422, June 2011.

[6] L.M.L. Ming, F. Sahlen, S. Halen, G. Brosig, and L. Palmqvist, "Impacts of high-frequency voltage on cable-terminations with resistive stressgrading," Proc. IEEE Int. Conf. Solid Dielectr. ICSD, vol. 1, no. 1, July 2004.

[7] V. Vapnik, "The Nature of Statistical Learning Theory," Springer, 1995.

[8] E.G. Ortiz-García,et al., "Accurate local very short-term temperature prediction based on synoptic situation Support Vector Regression banks," Atmospheric Research, Volume 107, pp. 1-8, April 2012.

[9] C. Chang and C. Lin. "LIBSVM: a Library for Support Vector Machines", April 2005, http://www.csie.ntu.edu.tw/ cjlin/libsvm.

[10] S. Christou, P. L. Lewin, J. A. Pilgrim, and S. G. Swingler, "The development of prognostic tools for MV cable circuits.", Electr. Insul. Conf. EIC, pp. 249-253, June 2014. 